\title{
Centered kNN Graph for Semi-Supervised Learning
}

\author{
Ikumi Suzuki \\ Yamagata University \\ Yonezawa, Yamagata, Japan \\ suzuki.ikumi@gmail.com
}

\author{
Kazuo Hara \\ National Institute of Genetics \\ Mishima, Shizuoka, Japan \\ kazuo.hara@gmail.com
}

\begin{abstract}
Graph construction is an important process in graph-based semisupervised learning. Presently, the mutual $k \mathrm{NN}$ graph is the most preferred as it reduces $h u b$ nodes which can be a cause of failure during the process of label propagation. However, the mutual $k \mathrm{NN}$ graph, which is usually very sparse, suffers from over sparsification problem. That is, although the number of edges connecting nodes that have different labels decreases in the mutual $k \mathrm{NN}$ graph, the number of edges connecting nodes that have the same labels also reduces. In addition, over sparsification can produce a disconnected graph, which is not desirable for label propagation. So we present a new graph construction method, the centered $k N N$ graph, which not only reduces hub nodes but also avoids the over sparsification problem.
\end{abstract}

\section{CCS CONCEPTS}

\section{- Information systems $\rightarrow$ Clustering and classification;}

\section{KEYWORDS}

graph-based SSL, mutual $k \mathrm{NN}$ graph, over sparsification problem

\section{INTRODUCTION}

Semi-supervised learning (SSL) is a class of supervised learning techniques that can make use of unlabeled data for training when a small amount of labeled data is available. SSL is known to work well even without label information if the unlabeled data can be used for finding data boundaries in a feature space, or if cluster or manifold structures can be recovered from the dataset.

One of the mainstream techniques for SSL follows a graph-based approach, wherein the samples are represented as nodes in a graph. The graph is constructed such that the manifold of the data is captured, and the information of labeled samples is propagated to target samples along the structure of the manifold, i.e., along the edges of the constructed graph, to predict their labels.

Graph-based SSL methods consist of two parts. (1) Constructing a graph without using label information, and (2) Propagating label information on the graph. In this paper, we focus on the first part. We present a new method to construct a graph that is appropriate for label propagation.

Permission to make digital or hard copies of all or part of this work for personal or classroom use is granted without fee provided that copies are not made or distributed for profit or commercial advantage and that copies bear this notice and the full citation on the first page. Copyrights for components of this work owned by others than ACM must be honored. Abstracting with credit is permitted. To copy otherwise, or republish, to post on servers or to redistribute to lists, requires prior specific permission and/or a

fee. Request permissions from permissions@acm.org.

SIGIR '17, August 07-11, 2017, Shinjuku, Tokyo, Japan.

(C) 2017 Association for Computing Machinery.

ACM ISBN 978-1-4503-4887-4/17/08 . \$15.00

https://doi.org/10.1145/3077136.3080662
For SSL to be efficient and robust to noise, sparsity of the graph is required [2]. A sparse graph commonly used for graph-based SSL is the $k$-nearest neighbor $(k \mathrm{NN})$ graph. However, the $k \mathrm{NN}$ graph likely contains $h u b$ nodes (i.e., nodes connected by many other nodes) and they affect label propagation, which is performed subsequently on the graph. Recently, Ozaki et al. [4] reported that the mutual kNN graph reduces the number of hub nodes, and de Sousa et al. [1] stated that the mutual $k \mathrm{NN}$ graph, at present, is the best choice for graph construction.

While the mutual $k \mathrm{NN}$ graph is considered state-of-the-art at present, we point out that the mutual $k \mathrm{NN}$ graph, which is usually a very sparse graph, suffers from over sparsification problem. Namely, although the number of edges connecting nodes that have different labels favorably decreases in the mutual $k \mathrm{NN}$ graph, the number of edges connecting nodes that have the same labels also reduces. In addition, over sparsification can produce a disconnected graph, which hinders label propagation on the graph.

In this paper, we present a new graph construction method, which not only reduces hub nodes but also solves the over sparsification problem. The new method, which constructs a graph called centered $k N N$ graph, does not remove edges from the $k \mathrm{NN}$ graph in order to reduce hub nodes as in the case of a mutual $k \mathrm{NN}$ graph. Instead, before constructing the $k \mathrm{NN}$ graph, the new method revises similarities between samples so that hubs are not produced, by using a technique known as centering. In the section of experiments, we demonstrate that, when using the centered $k \mathrm{NN}$ graph, the label propagation works well, and the accuracy of label prediction improves, compared with the mutual $k \mathrm{NN}$ graph.

\section{NOTATION OF GRAPH-BASED SSL}

Assume that we are given a set of $d$-dimensional feature vectors $\mathcal{X}=\left\{x_{i} \in \mathbb{R}^{d}\right\}_{i=1}^{n}$ with their labels $\mathcal{Y}=\left\{y_{i} \in S\right\}_{i=1}^{n}$, where $n$ is the number of samples and $S$ is a set of possible labels. Among the $n$ samples, we can only access to the labels $\left\{y_{i} \in S\right\}_{i=1}^{l} \subset \mathcal{Y}$ of $l$ samples (i.e., labeled data). In addition, a similarity or kernel function (including cosine similarity and Gaussian kernels) $f$ : $\mathbb{R}^{d} \times \mathbb{R}^{d} \mapsto \mathbb{R}$ is defined for a pair of samples, and a dense matrix $W$ of size $n \times n$ is given, where the its $i j$ th-component $W_{i j}=f\left(x_{i}, x_{j}\right)$.

More formally, we assume $f\left(x_{i}, x_{j}\right)=\left\langle\varphi\left(x_{i}\right), \varphi\left(x_{j}\right)\right\rangle$, i.e., the inner-product on a Hilbert space, where $\varphi(x)$ is a mapping function of a sample $x \in \mathcal{X}$ to the Hilbert space. Thus, $W_{i j}=\left\langle\varphi\left(x_{i}\right), \varphi\left(x_{j}\right)\right\rangle$.

The goal is to predict the labels $\left\{y_{i} \in S\right\}_{i=l+1}^{n} \subset \mathcal{Y}$ of $n-l$ samples (i.e., unlabeled data) which we cannot observe, using $\mathcal{X}$ and $\left\{y_{i} \in S\right\}_{i=1}^{l}$. To achieve this goal, the graph-based SSL constructs a weighted graph $\mathcal{G}$ from $\mathcal{X}$, where the $n$ samples correspond to the nodes in the graph. A construction of graph $\mathcal{G}$ is equivalent to a selection of an adjacency matrix $A$ that determines the weight of the edges in the graph $\mathcal{G}$. 


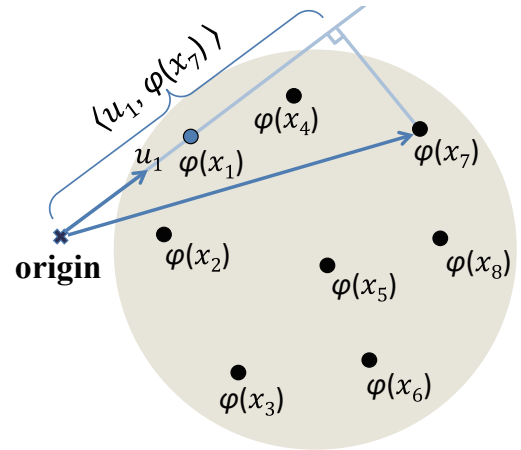

(a) An example dataset on a Hilbert space (an inner-product space)

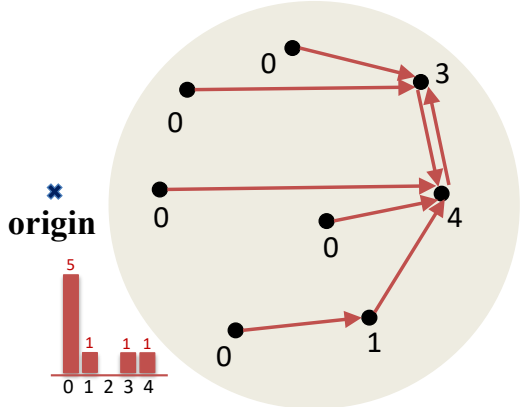

(b) Hubness occurs when the origin is away from the dataset center.

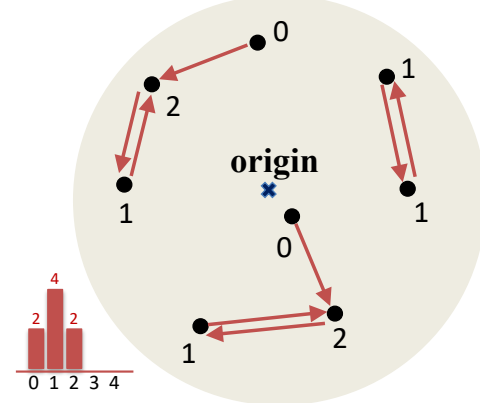

(c) Hubness disappears after we move the origin to the center.

Figure 1: An intuitive explanation of why the centering reduces hubs. (a)(b) When the origin is located on the left side of the dataset, most of the $1 \mathrm{NN}$ relations shown in red arrows tend to direct from left to right. Hence, some samples on the rightmost side become hubs with a large number of incoming arrows. (c) In contrast, when the origin is in the dataset center, the red arrows do not point in a certain direction.

Here, it seems that setting $A=W$ is the simplest choice. However, because similarity scores in $W$ for dissimilar sample pairs are often unreliable, using all the components of a dense matrix $W$ leads to low accuracy in predicting labels. Thus, it is desirable to construct a sparse graph from $W[2,4]$, and the most common one is the $k \mathrm{NN}$ graph.

In the following, we review existing sparse graphs used for graphbased SSL, and then, we will present the centered $k N N$ graph, which we consider appropriate for label propagation.

\section{EXISTING GRAPHS FOR SSL}

\subsection{The $k$ NN Graph}

The process to construct the $k \mathrm{NN}$ graph involves three steps. First, for a given similarity matrix $W$, the $k$ most similar samples for each of $n$ samples are determined, and a sparse binary matrix $V$ of size $n \times n$ is generated. Formally, $V_{i j}=1$ if the $i$ th sample is one of the $k$ most similar samples of the $j$ th sample according to the similarity scores in $W$, and $V_{i j}=0$ otherwise. Next, by using a max operation, the matrix $V$ is transformed into a symmetry matrix $U$, which is given by

$$
U=\max \left(V, V^{\mathrm{T}}\right) .
$$

Finally, a weighted adjacency matrix $A$ for the $k \mathrm{NN}$ graph is obtained for the $i j$ th component as

$$
A_{i j}=U_{i j} W_{i j}
$$

Note that, if the dimension $d$ of the feature space is high, the $k \mathrm{NN}$ graph constructed from $W$ tends to contain hub nodes that have an unexpectedly high degree (i.e., nodes connected by many other nodes), and the existence of hub nodes affects label propagation on the graph [4].

\subsection{The Mutual $k$ NN Graph}

The procedure to construct the mutual $k \mathrm{NN}$ graph is similar to that of the standard $k \mathrm{NN}$ graph. The difference lies in the way the matrix $V$ is symmetrized, which is carried out by

$$
U=\min \left(V, V^{\mathrm{T}}\right) \text {. }
$$

Note that any node in the mutual $k \mathrm{NN}$ graph has a degree (i.e., the number of edges that the node has) less than or equal to $k$, implying that hub nodes do not exist. Ozaki et al. [4] attributed the improvement in prediction accuracy when using the mutual $k \mathrm{NN}$ graph to this point.

On the other hand, we notice that the procedure to construct the mutual $k \mathrm{NN}$ graph involves removing edges from the standard $k \mathrm{NN}$ graph. In other words, the mutual $k \mathrm{NN}$ graph is constructed by sparsifying the standard $k \mathrm{NN}$ graph. Considering that the adjacency matrix $A$ of the standard $k \mathrm{NN}$ graph is obtained by sparsifying the similarity matrix $W$, the mutual $k \mathrm{NN}$ graph can be considered as a doubly-sparsified graph.

Although the mutual $k \mathrm{NN}$ graph is rendered free from hub nodes through double-sparsification, we point out that such strong sparsification not only removes harmful edges connecting nodes that have different labels, but also removes desirable edges connecting nodes that have same labels. Furthermore, over sparsification sometimes produces a disconnected graph, which affects label propagation. Therefore, in the next section, we present a method to construct a graph that contains few hub nodes but is not very sparse.

\section{THE CENTERED $K$ NN GRAPH}

Let $I$ be the identity matrix of size $n \times n$ and $\mathbb{1}$ be an $n$-dimensional all-ones vector. A centered similarity matrix is computed from $W$, in the form

$$
W^{\mathrm{CENT}}=\left(I-\frac{1}{n} \mathbb{1}^{\mathrm{T}}\right) W\left(I-\frac{1}{n} \mathbb{1}^{\mathrm{T}}\right) .
$$

Using the procedure described in Section 3.1, we generate a standard $k \mathrm{NN}$ graph from $W^{\mathrm{CENT}}$ instead of $W$. The resulting graph is called the centered kNN graph.

The purpose of the centering operation in Equation (3) is to reduce hubs. We provide an intuitive explanation by using a small 


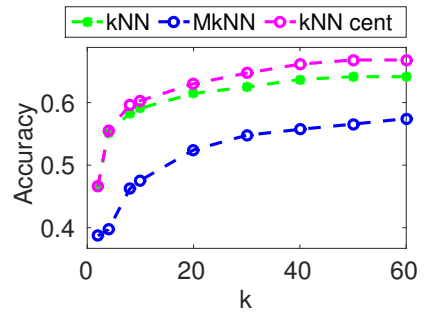

(a) Mini Newsgroups

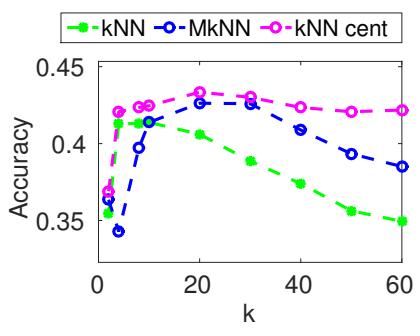

(b) Reuters Transcribed

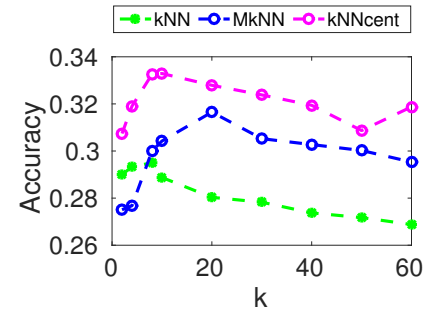

(c) WSD

Figure 2: Accuracy of label prediction using the standard (kNN), mutual (MkNN), and centered (kNN cent) $k$ NN graphs for varying size of $k$.

dataset $D=\left\{x_{1}, \ldots, x_{8}\right\}$ consisting of eight samples. Figure 1(a) shows the dataset mapped onto a Hilbert space as $\left\{\varphi\left(x_{1}\right), \ldots, \varphi\left(x_{8}\right)\right\}$. To construct the $k \mathrm{NN}$ graph, we find the $k$ most similar samples for each sample $x \in D$. Similarity is measured by the inner-product on the Hilbert space.

For the standard $k \mathrm{NN}$ graph, when $k=1$, the sample most similar to $x_{i}$, denoted by $1 \mathrm{NN}\left(x_{i}\right)$, is determined as

$$
1 \mathrm{NN}\left(x_{i}\right)=\underset{x \in D \backslash\left\{x_{i}\right\}}{\operatorname{argmax}}\left\langle\varphi\left(x_{i}\right), \varphi(x)\right\rangle=\underset{x \in D \backslash\left\{x_{i}\right\}}{\operatorname{argmax}}\left\langle u_{i}, \varphi(x)\right\rangle,
$$

where $u_{i}$ is the unit vector of $\varphi\left(x_{i}\right)$. That is, the sample most similar to $x_{i}$ is the sample $x \in D \backslash\left\{x_{i}\right\}$ such that the mapped vector $\varphi(x)$ has the largest component of the vector $u_{i}$. Thus, in Figure 1(a), $1 \mathrm{NN}\left(x_{1}\right)=x_{7}$.

Figure 1(b) shows a directed graph where each sample $x_{i}$ is connected to $1 \mathrm{NN}\left(x_{i}\right)$ by a red arrow. Note that if we transform it to an undirected graph (i.e., ignore the direction of the arrows), we obtain the standard $k \mathrm{NN}$ graph.

We note that there is a common direction in the arrows in Figure 1(b): most of the arrows tend to direct from the origin to the data center, i.e., from left to right in this case. Consequently, a few samples with a large number of incoming arrows, i.e., hub samples, emerge on the side right to the origin. In Figure 1(b), the number attached to a sample denotes the number of incoming arrows to the sample (i.e. the reverse $k \mathrm{NN}$ count). Moreover, their histogram is shown. The histogram is not symmetric (it is skewed to the right), and this indicates the occurrence of hubness [5].

In contrast, the centered $k \mathrm{NN}$ graph is obtained using Equation (3), equivalently by moving the origin to the data center $c=$ $\frac{1}{|D|} \sum_{x^{\prime} \in D} \varphi\left(x^{\prime}\right)$, as in Figure 1(c). We notice that the red arrows in Figure 1(c) do not have a common direction (i.e., we obtain more scattered $k$ nearest neighbors) and the histogram becomes symmetric. This implies that hubness is reduced, at least for single-cluster data. $^{1}$

Notably, in contrast with the mutual $k \mathrm{NN}$ graph, the centered $k \mathrm{NN}$ graph is not very sparse. This is because the max operation in Equation (1) is used, rather than the min operation in Equation (2). Consequently, the sparsity level of the centered $k \mathrm{NN}$ graph is the same as that of the standard $k \mathrm{NN}$ graph.

\footnotetext{
${ }^{1}$ For multi-cluster data, we may consider more than one origins for different clusters
}

\section{EXPERIMENTS}

We compared the centered $k \mathrm{NN}$ graph with the state-of-the-art mutual $k \mathrm{NN}$ graph, as well as the standard $k \mathrm{NN}$ graph as a baseline. We evaluated the accuracy of label prediction in two multi-class classification tasks: document classification and word sense disambiguation (WSD).

Datasets and SSL Settings: For the document classification task, we used the Reuters Transcribed and Mini Newsgroups datasets. ${ }^{2}$ The number of documents (i.e., $n$ ) is 201 and 2000, and the number of classes (i.e., $|S|$ ) is 10 and 20 , respectively.

For the WSD task, we used the data for the Senseval-3 ELS task, which is a collection of 57 datasets for different polysemous words [3]. Averaged over 57 datasets, 207.1 occurrences $(n=207.1)$ of a target word are annotated from 6.4 gold standard senses $(|S|=6.4)$ of the target word. For each dataset, we created a tf-idf weighted vector for each occurrence of the target word from its surrounding context, and we computed $W$ using cosine similarity.

After constructing the standard, mutual, and centered $k \mathrm{NN}$ graphs using the similarity matrix $W$, we ran the Local and Global Consistency [6], one of the state-of-the-art label propagation algorithms, with a smoothing parameter $\mu=0.9$, following [4].

To simulate SSL in which only a small amount of labeled data is available, we selected $l$ samples from the original dataset and used these as labeled data, where $l$ was $5,10,15$, or $20 \%$ of the total number of samples. ${ }^{3}$ The remaining $n-l$ samples were used as unlabeled data, whose labels form the prediction target. For each dataset, we randomly selected $l$ samples 10 times, and reported the results obtained by taking the average over all trials.

Results: The label prediction accuracy is compared for the Mini Newsgroups, Reuters Transcribed, and WSD datasets, in Figure 2(a), 2(b) and 2(c), respectively. Because the WSD data consists of 57 datasets, the averaged accuracy over all datasets is displayed in Figure 2(c). These figures show that in most cases, the centered $k \mathrm{NN}$ graph achieves better accuracy than the other graphs.

\footnotetext{
2 https://archive.ics.uci.edu/ml

${ }^{3}$ Because the same trend was observed, only the figures when $l=0.10 \times n$ are displayed in Figure 2.
} 


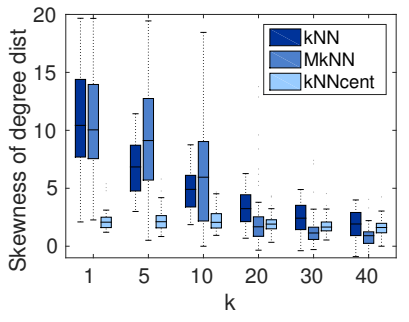

(a) Hubness

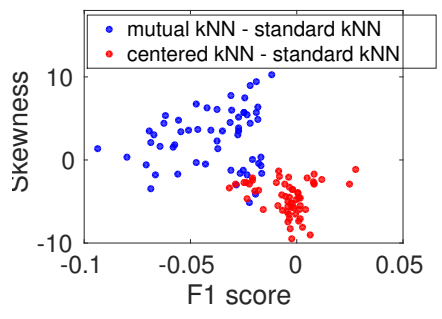

(b) Hubness and F1
Figure 3: (a) Hubness measured by the skewness of node degree distribution in the standard, mutual, and centered $k \mathrm{NN}$ graphs computed for WSD datasets. (b) A scatter plot of 57 WSD datasets for comparison between the mutual and centered $k \mathrm{NN}$ graphs (i.e., difference of skewness and F1 score against the standard $k \mathrm{NN}$ graph, when $k=5$ ).
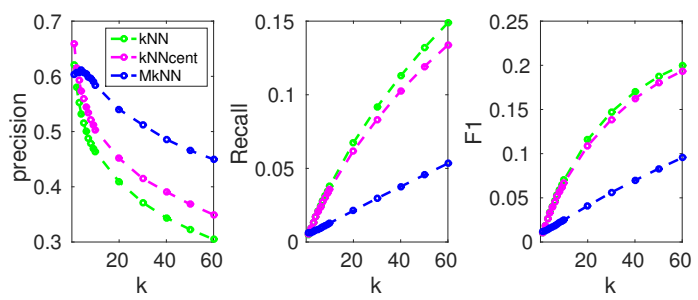

(a) Mini Newsgroups
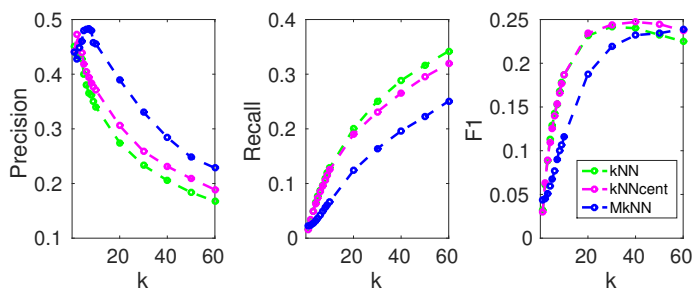

(b) Reuters Transcribed
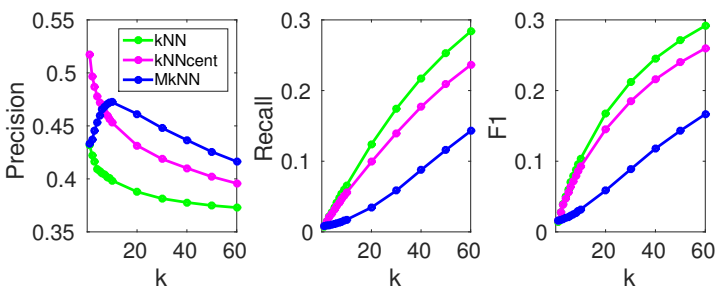

(c) WSD

Figure 4: Precision, Recall and F1 score for the correct edges in the standard $(\mathrm{kNN})$, mutual $(\mathrm{MkNN})$, and centered $(\mathrm{kNN}$ cent) $k \mathrm{NN}$ graphs.

\section{DISCUSSION}

We now discuss why the centered $k \mathrm{NN}$ graph performed better in these experiments, by examining the quality of the constructed graphs in terms of two properties: hubness, and the F1 score regarding correct edges. ${ }^{4}$

First, we evaluate the emergence of hub nodes, which affects label propagation and hence deteriorates the performance, by measuring the skewness of the node degree (i.e., the number of edges incident to a node) distribution. A large skewness indicates strong hubness [5].

For the WSD data, the skewness is shown in Figure 3(a). We have computed the skewness for each of the 57 datasets in the WSD data, and have presented the distribution of the skewness in the form of box plots. Figure 3(a) shows that the centered $k \mathrm{NN}$ graph successfully reduces the hub nodes. However, as for the mutual $k \mathrm{NN}$ graph, skewness tends to increase compared to the standard $k \mathrm{NN}$ graph when $k$ is small (i.e., $k \leq 10$ ). This is because, owing to the over sparsification, the mutual $k \mathrm{NN}$ graph often contains small disconnected components including isolated nodes, and the hubness appears as a result of creating a connected graph using the maximum spanning tree algorithm [4].

Next, we discuss the reason behind the occasional drop in accuracy of the mutual $k \mathrm{NN}$ graph, as in the case of Mini Newsgroups in Figure 2(a). For each of the $k \mathrm{NN}$ graphs, we counted the number of correct edges connecting samples bearing the same labels. We then calculated its ratio to the number of edges in the $k \mathrm{NN}$ graph (i.e., precision), and also calculated its ratio to the number of correct edges in the complete graph where every pair of samples is connected (i.e., recall). Figure 4 shows the precision, recall, and F1 scores for the correct edges in the standard, mutual, and centered $k \mathrm{NN}$ graphs. The figure indicates that although precision is improved in the mutual $k \mathrm{NN}$ graph, recall is considerably deteriorated owing to the over sparsification of the graph. As a result, the F1 score for the correct edges could become very small, and hence, it can be considered that the mutual $k \mathrm{NN}$ graph makes the accuracy worse for the Mini Newsgroups.

In contrast, the centered $k \mathrm{NN}$ graph reduces hub nodes without changing F1 score for correct edges significantly, which can be considered a desirable property for successful label propagation.

\section{ACKNOWLEDGMENTS}

We would like to thank anonymous reviewers for their helpful comments. This work was supported by JSPS KAKENHI Grant Numbers JP16K00066 and JP16H02821.

\section{REFERENCES}

[1] Celso Andre Rodrigues de Sousa, Solange Oliveira Rezende, and Gustavo EnriqueAlmeidaPradoAlves Batista. 2013. Influence of Graph Construction on Semi-supervised Learning. In ECML/PKDD. 160-175.

[2] Tony Jebara, Jun Wang, and Shih-Fu Chang. 2009. Graph Construction and B-matching for Semi-supervised Learning. In ICML. 441-448.

[3] Rada Mihalcea, Timothy Chklovski, and Adam Kilgarriff. 2004. The Senseval-3 English lexical sample task. In Senseval-3. 25-28.

[4] Kohei Ozaki, Masashi Shimbo, Mamoru Komachi, and Yuji Matsumoto. 2011. Using the Mutual k-Nearest Neighbor Graphs for Semi-supervised Classification on Natural Language Data. In CoNLL. 154-162.

[5] Miloš Radovanović, Alexandros Nanopoulos, and Mirjana Ivanović. 2010. On the existence of obstinate results in vector space models. In SIGIR. 186-193.

[6] Dengyong Zhou, Olivier Bousquet, Thomas Navin Lal, Jason Weston, and Bernhard Schölkopf. 2004. Learning with local and global consistency. In NIPS. 595-602.

\footnotetext{
${ }^{4}$ Due to lack of space, we have not shown the skewness for the document classification tasks, in which both the centered and mutual $k \mathrm{NN}$ graphs have reduced hubness.
} 